\title{
Is a train-the-trainer method of teaching an effective way of implementing the correct use of the 'Malnutrition Universal Screening Tool' ('MUST') in care homes?
}

\author{
R. Lee and F. Scott \\ Department of Nutrition \& Dietetics, Norfolk and Norwich University Hospitals NHS Foundation Trust, Colney Lane, \\ Norwich NR4 7UY, UK
}

Following a successful 'MUST' training programme in six Social Services care homes ${ }^{(1)}$ Norfolk County Council Adult Social Services funded the development and delivery of a train-the-trainer teaching package, as a realistic way of implementing 'MUST' in all Norfolk care homes.

Seven courses were held in 2007 to train care-home staff how to teach their colleagues to use the 'MUST'. A course comprised $1 \mathrm{~d}$ formal teaching, followed by an assessment of the new trainer delivering a teaching session. Delegates were provided with a dossier of support materials to use when training.

Data were collected before and 3 months after the course on: frequency of nutritional screening; provision of suitable foods and drinks for snacks; fortification of meals. The delegates were also asked to evaluate the course for its comprehensibility and usefulness in preparing them to teach. In addition, their level of understanding and ability to calculate a 'MUST' score accurately and devise suitable care plans were assessed during the training day and via the checking of sample care plans from their workplaces 3 months after the course.

Sixty-seven delegates were trained, of which $44 \%$ completed and returned follow-up questionnaires and provided copies of sample care plans. The majority of care plans showed that steps 1-4 of the 'MUST' were being calculated accurately (although $12 \%$ had inaccurate calculations of $\%$ weight loss) and that appropriate treatment care plans had been set up, evaluated and developed.

Table 1. Percentage accuracy in calculating steps $1-4$ of 'MUST'

\begin{tabular}{|c|c|c|c|c|c|c|c|c|}
\hline \multicolumn{3}{|c|}{ Calculation of BMI $(n$ 167) } & \multicolumn{3}{|c|}{ Calculation height from ulna length $(n$ 167) } & \multicolumn{3}{|c|}{ Calculation of $\%$ weight loss $(n 167)$} \\
\hline Correct & N/A & Incorrect & Correct & N/A & Incorrect & Correct & N/A & Incorrect \\
\hline 93 & 5 & 2 & 47 & 47 & 6 & 83 & 5 & 12 \\
\hline
\end{tabular}

Table 2. Percentage accuracy in provision of appropriate care plans

\begin{tabular}{|c|c|c|c|c|c|c|c|c|}
\hline \multicolumn{3}{|c|}{ Appropriate care plan set up $(n 167)$} & \multicolumn{3}{|c|}{ Appropriate care plan evaluation $(n$ 167) } & \multicolumn{3}{|c|}{ Appropriate development $(n$ 167) } \\
\hline$\overline{\text { Yes }}$ & N/A & No & Yes & N/A & No & Yes & N/A & No \\
\hline$\overline{93}$ & 0 & 7 & 87 & 9 & 4 & 84 & 6 & 10 \\
\hline
\end{tabular}

Results showed that regular nutrition screening increased from $42 \%$ (nine of which were already using the 'MUST') at baseline to $96 \%$ (all using the 'MUST') at 3 months. The use of full-fat milk increased from $60 \%$ of care homes to $80 \%$ and provision of snacks increased, as did the variety of foods offered.

In total $100 \%$ of the new trainers rated the course as effective or very effective in preparing them to deliver training and $96 \%$ were assessed when delivering training as competent in explaining the 'MUST' accurately.

The train-the-trainer model of teaching is an effective method of training care-home staff in the correct use of the 'MUST'. However, the miscalculation of $\%$ weight loss in $12 \%$ of the care plans suggested that more training time needs to be spent on this aspect. In addition, assessments of each new trainer delivering training were very time consuming for the authors. Given the accuracy in explaining the 'MUST', this aspect might be omitted in future.

1. Merriman SH, Scott HF, Paterson KE et al. (2008) Proc Nutr Soc 67, E131. 